${ }^{1}$ Universität Bremen, Institut für Public Health und Pflegeforschung

2 Universität Bremen, Zentrum für Klinische Psychologie und Rehabilitation

Karl Reif' (Prof. Dr.), Ulrike de Vries² (Dr. phil.), Franz Petermann² (Prof. Dr.)

\title{
Was hilft wirklich bei tumorbedingter Fatigue? Ein Überblick über systematische Übersichtsarbeiten
}

- Was ist (zu dieser Thematik) schon bekannt?

Fatigue ist ein häufiges und belastendes Symptom bei Krebspatient(inn)en, für das vielfältige Interventionen vorgeschlagen werden.

- Was ist neu?

In der vorliegenden Arbeit wird ein Überblick über die Wirksamkeit von nichtpharmakologischen Interventionen bei tumorbedingter Fatigue gegeben.

- Welche Konsequenzen haben die Ergebnisse für die Pflegepraxis? Bewegungsförderung und psychosoziale Unterstützung sollten in der pflegerischen Anleitung, Beratung und Schulung von Krebspatient(inn)en mit Fatigue sowie in der Koordination der Betreuung stärker berücksichtigt werden.

Tumorbedingte Fatigue, ein subjektives Gefühl von ausgeprägter physischer, emotionaler und/oder kognitiver Erschöpfung, ist ein häufiges Phänomen bei Patient(inn)en mit zytotoxischer Chemotherapie oder Strahlentherapie. Die Forschung über tumorbedingte Fatigue wurde im vergangenen Jahrzehnt intensiviert. In der vorliegenden Arbeit wurde das Ziel verfolgt, einen Überblick über die Wirksamkeit von nichtpharmakologischen Interventionen zu tumorbedingter Fatigue zu geben. Hierzu wurden die Datenbanken der Cochrane Library, PubMed, CINAHL und DAHTA nach systematischen Übersichtsarbeiten durchsucht. Die Auswahl und Bewertung der methodischen Qualität der Reviews erfolgte unabhängig durch zwei Personen. Zur Bewertung wurde das Instrument AMSTAR verwendet. Die Synthese der Ergebnisse erfolgte qualitativ

Manuskript erstmals eingereicht am 21.04.2012 Endgültige Fassung eingereicht am 25.09.2012 in Form einer tabellarischen Übersicht. Insgesamt wurden 24 Reviews in die Analyse eingeschlossen. Die Ergebnisse zeigen, dass körperliche Bewegung und psychosoziale Unterstützung die Fatiguebelastung von Krebspatient(inn)en reduzieren können. Insbesondere zeigten sich von Pflegekräften durchgeführte, fatiguespezifische Interventionen als wirksam. Für weitere Interventionsformen ist derzeit die Evidenz schwach, gleichwohl es vielversprechende Ansätze gibt. Trotz einer hohen Zahl an randomisierten klinischen Studien wie auch systematischen Übersichtsarbeiten ist die Studienlage noch an vielen Stellen defizitär. Die Ergebnisse können von der Pflege für die klinische Betreuung, Beratung und Schulung von Krebspatient(inn)en genutzt werden.

Schlüsselwörter: Fatigue, Krebs, systematische Übersichtsarbeit, onkologische Pflege, Pflegeforschung

\section{Einleitung}

Tumorbedingte Fatigue ist ein anhaltendes, subjektives Gefühl von körperlicher, emotionaler und/oder kognitiver Müdigkeit beziehungsweise Erschöpfung, das im Rahmen einer Krebserkrankung oder Tumorbehandlung ohne Zusammenhang mit vorheriger Beanspruchung auftritt und die Alltagsfunktionalität sowie die Lebensqualität beeinträchtigt (National Comprehensive Cancer Network (NCCN), 2012). Insbesondere Patient(inn)en, die zytotoxische Chemotherapie, Strahlentherapie, Knochenmark- beziehungsweise Stammzelltransplantation und/ oder Biologicals erhalten, leiden erheblich unter Fatigue. Zum Zeitpunkt der Diagnose leiden etwa $40 \%$ der $\mathrm{Pa}$ tient(inn)en unter Fatigue. Nahezu alle Patient(inn)en erleben Müdigkeit während der Krebstherapie: bis zu 90\% der Patient(inn)en mit Strahlentherapie und bis zu $80 \%$ der Patient(inn)en mit Chemotherapie. Fatigue tritt noch Monate oder sogar Jahre nach Abschluss der Behandlung bei etwa einem Drittel der Patient(inn)en mit Krebs auf (Hofman, Ryan, Figueroa-Moseley, JeanPierre \& Morrow, 2007).

Fatigue hat einen erheblichen negativen Einfluss auf den Gesundheitszustand, das Befinden, den Krankheitsverlauf, die Lebensqualität, die Alltagsbewältigung und die ökonomische Situation von Krebspatient(inn)en (de Vries, Reif \& Petermann, 2011a; de Vries, Reif \& Petermann, 2011b; de Vries, Reif, Stuhldreher, Petermann \& Görres, 2009). Pflegekräfte kommen in der Betreuung von Krebspatient(inn)en mit allen Aspekten der Fatigue in der Akutversorgung, in der Langzeitbetreuung wie auch in der Palliativsituation in Kontakt. Daher ist es für die Pflege in der Betreuung, Beratung und Schulung von Krebspatient(inn)en elementar, 
einen umfassenden Überblick über die Interventionsmöglichkeiten bei Fatigue zu gewinnen (Reif, 2012).

Patient(inn)en erleben Fatigue als belastender als andere Nebenwirkungen, die mit der Tumorerkrankung oder Therapie einhergehen, etwa Schmerzen, Übelkeit und Erbrechen (Stone, Richards, A'Hern \& Hardy, 2000). Bei Gesunden tritt Erschöpfung nach erfolgter körperlicher oder kognitiver Anstrengung auf, und die Erholung findet durch Ausruhen und Schlaf statt; von Fatigue betroffene Patient(inn)en erfahren jedoch eine Erschöpfung, die unabhängig von vorheriger Anstrengung auftreten und durch Ausruhen und Schlaf kaum beeinflusst werden kann.

Als mögliche Ursachen und prädisponierende Faktoren für tumorbedingte Fatigue werden immunologische, endokrine, neurologische, psychologische, hämatologische muskuloskelettale und Ernährungsfaktoren diskutiert (Patarca-Montero, 2004). Darüber hinaus können auch der Abbau der Skelettmuskulatur durch Immobilität (Al-Majid \& McCarthy, 2001) und Störungen des zirkadianen Rhythmus (Berger, Wielgus, Hertzog, Fischer \& Farr, 2010) zur Entstehung von Fatigue beitragen. Diese ätiologischen Mechanismen werden bislang lediglich durch eine schwache Evidenz gestützt. Fatigue wird heute als komplexes, multifaktoriell bedingtes Phänomen betrachtet.

\section{Fragestellung und Ziel der Arbeit}

Die wissenschaftliche Literatur zu Interventionen bei Fatigue ist mittlerweile fast unüberschaubar. Der jährliche $\mathrm{Zu}$ wachs an Beiträgen zu tumorbedingter Fatigue beträgt in der Datenbank Medline über 600 Publikationen. Schon die Suche nach Reviews ergibt eine kaum überschaubare Fülle an Veröffentlichungen. Daher wurde in der vorlie- genden Arbeit das Ziel verfolgt, einen Überblick über die systematischen Übersichtsarbeiten zu nichtpharmakologischen Interventionen bei tumorbedingter Fatigue zu geben.

Liegen mehrere Übersichtsarbeiten vor, so bietet ein Overview of Reviews einen schnellen, umfassenden Überblick über Interventionen zu einem bestimmten Problem (Pflegephänomen, Symptom beziehungsweise Symptomkomplex, Erkrankung etc.). Overviews können verschiedene Ziele verfolgen (Becker \& Oxman, 2011); die vorliegende Arbeit verfolgte das Ziel, Evidenz aus mehreren Übersichtsarbeiten $\mathrm{zu}$ verschiedenen Interventionen bei tumorbedingter Fatigue zusammenzufassen. Das Nachrecherchieren neuerer Primärstudien in Form eines Updates von Übersichtsarbeiten wird generell nicht empfohlen (Becker \& Oxman 2011), stattdessen sollte vor allem bei veralteten Übersichten ein neuer Review von Primärstudien in Betracht gezogen werden. Dies ginge über die Ziele eines Overviews hinaus, wäre jedoch für einen Health Technology Report oder eine evidenzbasierte Leitlinie ein angemessenes Vorgehen.

Ausgangssituation für die Arbeit war die Entwicklung und Evaluation eines Selbstmanagementprogramms für Krebspatient(inn)en mit Fatigue (Stuhldreher, Reif, de Vries, Görres \& Petermann, 2008; Reif, de Vries, Petermann \& Görres, 2010; Reif, de Vries, Petermann \& Görres, 2012).

\section{Methoden}

Es wurde eine systematische Übersicht über systematische Übersichtsarbeiten (Smith, Devane, Begley \& Clarke, 2011) von Interventionen zur Bewältigung von Fatigue bei Krebspatient(inn)en in allen Krankheitsstadien erstellt.

Hierzu wurden die Datenbanken Cochrane Database of Systematic Reviews (CDSR), Cochrane Database of Abstracts of Reviews of Effects (DARE), Cochrane Health Technology Assessment Database (HTA), Medline, CINAHL, sowie die DAHTA-Datenbank des Deutschen Instituts für Medizinische Dokumentation und Information (DIMDI) verwendet. Die Suchstrategien (Tabelle 1) wurden bewusst breit angelegt, um möglichst wenig Information zu übersehen. Zudem wurden die Referenzlisten der im Volltext durchgearbeiteten Reviews systematisch durchsucht.

Eingeschlossen wurden alle systematischen Reviews, die Interventionen bei tumorbedingter Fatigue zum Gegenstand hatten. Ausgeschlossen wurden zunächst bei der Auswahl nach Titel (1. Schritt) und Abstract (2. Schritt) alle narrativen Reviews, Beiträge, die keine Reviews waren, Beiträge, die sich auf Arzneimitteltherapien bezogen, sowie Reviews, die keine Krebspatient(inn)en eingeschlossen hatten. Der Ausschluss von Volltexten (3. Schritt) erfolgte aus den oben genannten Gründen und zusätzlich, wenn sich herausstellte, dass

Tabelle 1: Suchstrategien und -begriffe.

\begin{tabular}{|ll|}
\hline CDRS & fatigue \\
DARE & fatigue \\
HTA & fatigue \\
Medline & systematic[sb] AND («Neoplasms»[Mesh] AND fatigue) \\
CINAHL & fatigue, Limiters: klinische Fragen: Therapy - High Sensitivity; \\
& Publikationstyp: Systematic Review; spezielles Interesse: \\
& Oncologic Care \\
DAHTA-Datenbank & fatigue \\
\hline
\end{tabular}


die Arbeit überholt war. Das hieß, dass neuere Publikationen vorlagen, welche die vorherigen umfassten, dass eine Aktualisierung des Reviews vorlag, oder dass Teilergebnisse eines bereits eingeschlossenen Reviews enthalten waren. Die Auswahl wurde nicht auf Reviews von randomisiert-kontrollierten Studien (RCTs) beschränkt, da etliche nichtmedikamentöse Maßnahmen nicht in RCTs geprüft wurden. Durch einen Verzicht auf Non-RCTs würde ein Teil der vorhandenen Evidenz nicht erfasst, wenn dafür auch ein höheres Risiko für Bias in Kauf genommen werden musste. Es fanden keine Einschränkungen hinsichtlich des Jahres der Publikation und der Sprache statt.

Die Auswahlstrategie der Reviews wird entsprechend des Preferred Reporting Items for Systematic Reviews and Meta-Analyses (PRISMA)-Statements (Liberati, Altman, Tetzlaff, Mulrow, Gøtzsche, Ioannidis et al., 2009) dargestellt. Obwohl PRISMA für Reviews von randomisierten Studien entwickelt wurde, ist es grundlegend auch geeignet, die Auswahl systematischer Übersichten darzustellen.

Zur Bewertung der methodischen Qualität wurde AMSTAR (Shea, Grimshaw, Wells, Boers, Andersson, Hamel et al., 2007a) verwendet, ein reliables und valides Assessmentinstrument (Shea, Bouter, Peterson, Boer, Andersson, Ortiz et al., 2007b; Shea, Hamel, Wells, Bouter, Kristjansson, Grimshaw et al., 2009) mit insgesamt 11 Items. Jedes Item kann mit «Ja», «Nein», «nicht beantwortbar» oder «nicht zutreffend» beantwortet werden.

Die Auswahl und Bewertung der eingeschlossenen Reviews erfolgte unabhängig voneinander durch zwei Personen (KR und UdV). Diskrepanzen wurden bis zum Konsens diskutiert.

Die Synthese der Ergebnisse erfolgte qualitativ in Form einer tabellarischen Übersicht der Geltungsbereiche, Charakteristika, wichtigsten Ergebnisse und methodischen Bewertung aller eingeschlossenen Arbeiten.

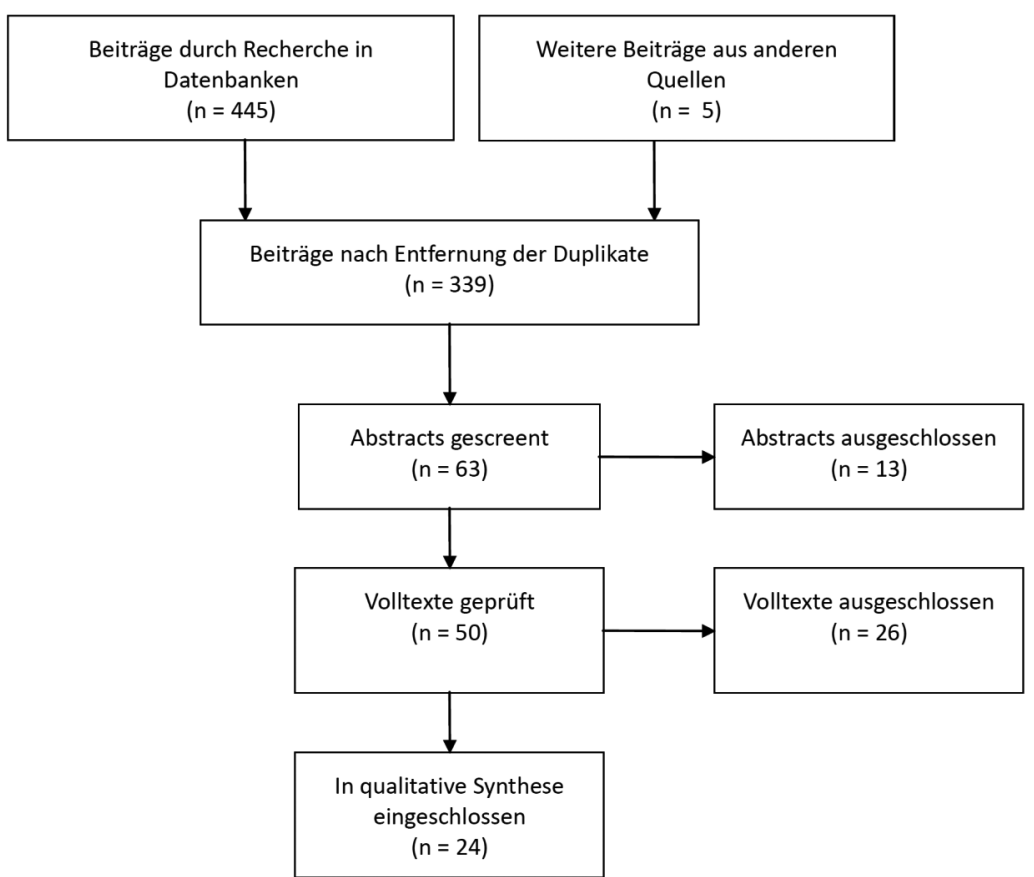

Abbildung 1: Flussdiagramm.

\section{Ergebnisse}

Insgesamt wurden 445 Einträge in den Datenbanken und 5 Beiträge aus den Referenzlisten gefunden. Die Aufteilung der Beiträge auf die verschiedenen Datenbanken zeigt Tabelle 2 .

Nach Entfernung der Duplikate blieben noch 339 Beiträge, deren Titel gescreent wurden. Nach Ausschluss irrelevanter Titel blieben 63 Beiträge. Von diesen wurden 13 irrelevante Beiträge ausgeschlossen, so dass 50 Beträge im Volltext geprüft wurden. Hiervon wurden 26 Arbeiten ausgeschlossen (Tabelle 3) und 24 in den vorliegenden Overview eingeschlossen (Tabellen 4 und 5). Der Verlauf der Suchergebnisse wird im Flussdiagramm (Abbildung 1) dargestellt.

Die gefundenen Übersichtsarbeiten reichten bis in das Jahr 1996 zurück. Für den Einschluss von Reviews war keine grundsätzliche Jahresgrenze vorgegeben, dennoch zeigte sich bei der Durchsicht der Volltexte, dass die Ergebnisse älterer Reviews oft in den neueren Übersichtsarbeiten ergänzt oder revidiert wurden. Meist fanden zudem in jüngeren Übersichtsarbeiten strengere methodische Einschlusskriterien als in älteren Arbeiten statt.

Übersichtsarbeiten, die sehr allgemein das Management von Fatigue zum Ziel hatten, wurden zum Teil nach Durch-

Tabelle 2: Anzahl Beiträge pro Datenbank.

\begin{tabular}{|ll|}
\hline Datenbank & $\begin{array}{l}\text { Anzahl der } \\
\text { Beiträge }\end{array}$ \\
CDSR & 105 \\
DARE & 51 \\
HTA & 23 \\
Medline & 234 \\
CINAHL & 31 \\
DAHTA-Datenbank & 1 \\
gesamt & 445 \\
\hline
\end{tabular}


Tabelle 3: Nach Durchsicht der Volltexte ausgeschlossene Literatur.

\begin{tabular}{|c|c|}
\hline Autor(inn)en und Jahr & Titel und Zeitschrift \\
\hline Barsevick, A. M.; Newhall, T. \& Brownm, S. (2008). & $\begin{array}{l}\text { Management of cancer-related fatigue. Clinical Journal of } \\
\text { Oncology Nursing, } 12 \text { (5 Suppl), 21-25. }\end{array}$ \\
\hline $\begin{array}{l}\text { Bohlius, J.; Schmidlin, K.; Brillant, C.; Schwarzer, G.; Trelle, S.; } \\
\text { Seidenfeld, J.; Zwahlen, M.; Clarke, M. J.; Weingart, O.; Kluge, S.; } \\
\text { Piper, M.; Napoli, M.; Rades, D.; Steensma, D.; Djulbegovic, B.; } \\
\text { Fey, M. F.; Ray-Coquard, I.; Moebus, V.; Thomas, G.; Untch, M.; } \\
\text { Schumacher, M.; Egger, M. \& Engert, A. (2009). }\end{array}$ & $\begin{array}{l}\text { Erythropoietin or Darbepoetin for patients with cancer- } \\
\text { meta-analysis based on individual patient data. Cochrane } \\
\text { Database of Systematic Reviews 2009, Issue 3. Art. No.: } \\
\text { CD007303. DOI: 10.1002/14651858.CD007303.pub2. }\end{array}$ \\
\hline $\begin{array}{l}\text { Bohlius, J.; Wilson, J.; Seidenfeld, J.; Piper, M.; Schwarzer, G.; } \\
\text { Sandercock, J.; Trelle, S.; Weingart, O.; Bayliss, S.; Brunskill, S.; } \\
\text { Djulbegovic, B.; Bennett, C.; Langensiepen, S.; Hyde, C. \& } \\
\text { Engert, A. (2006). }\end{array}$ & $\begin{array}{l}\text { Erythropoietin or Darbepoetin for patients with cancer. } \\
\text { Cochrane Database of Systematic Reviews 2006, Issue 3. Art. } \\
\text { No.: CD003407. DOI: 10.1002/14651858.CD003407.pub4. }\end{array}$ \\
\hline $\begin{array}{l}\text { Carr, D.; Goudas, L.; Lawrence, D.; Pirl, W.; Lau, J.; DeVine, D.; } \\
\text { Kupelnick, B. \& Miller, K. (2002). }\end{array}$ & $\begin{array}{l}\text { Management of cancer symptoms: pain, depression, and } \\
\text { fatigue. Evidence Report - Technology Assessment (Summary), } \\
61,1-5 .\end{array}$ \\
\hline $\begin{array}{l}\text { Carroll, J. K.; Kohli, S.; Mustian, K.M.; Roscoe, J. A. \& Morrow, } \\
\text { G. R. (2007). }\end{array}$ & $\begin{array}{l}\text { Pharmacologic treatment of cancer-related fatigue. } \\
\text { Oncologist, } 12 \text { (Suppl 1), } 43-51 .\end{array}$ \\
\hline Courneya, K. S. \& Friedenreich, C. M. (1999). & $\begin{array}{l}\text { Physical exercise and quality of life following cancer diagno- } \\
\text { sis: a literature review. Annals of Behavioral Medicine, } 21 \text { (2), } \\
171-179 .\end{array}$ \\
\hline Eton, D. T. \& Cella, D. (2011). & $\begin{array}{l}\text { Do erythropoietic-stimulating agents relieve fatigue? A review } \\
\text { of reviews. Cancer Treatment and Research, 157, 181-94. }\end{array}$ \\
\hline $\begin{array}{l}\text { Fong, D. Y.; Ho, J. W.; Hui, B. P.; Lee, A. M.; Macfarlane, D.J.; } \\
\text { Leung, S. S.; Cerin, E.; Chan, W. Y.; Leung, I. P.; Lam, S. H.; } \\
\text { Taylor, A. J. \& Cheng, K. K. (2012). }\end{array}$ & $\begin{array}{l}\text { Physical activity for cancer survivors: meta-analysis of } \\
\text { randomised controlled trials. British Medical Journal, 344, e70. }\end{array}$ \\
\hline $\begin{array}{l}\text { Foster, G.; Taylor S. J. C.; Eldridge, S.; Ramsay, J. \& } \\
\text { Griffiths, C. J. (2007). }\end{array}$ & $\begin{array}{l}\text { Self-management education programmes by lay leaders for } \\
\text { people with chronic conditions. Cochrane Database of } \\
\text { Systematic Reviews: Reviews 2007 Issue } 4 \text { John Wiley \& Sons, } \\
\text { Ltd Chichester, UK DOI: 10.1002/14651858.CD005108.pub2 }\end{array}$ \\
\hline Friedenreich, C. M. \& Courneya, K. S. (1996). & $\begin{array}{l}\text { Exercise as rehabilitation for cancer patients. Clinical Journal } \\
\text { of Sport Medicine, } 6 \text { (4), 237-244. }\end{array}$ \\
\hline $\begin{array}{l}\text { Galantino, M. L.; Cannon, N.; Hoelker, T.; Iannaco, J. \& } \\
\text { Quinn, L. (2007). }\end{array}$ & $\begin{array}{l}\text { Potential benefits of walking and yoga on perceived levels of } \\
\text { cognitive decline and persistent fatigue in women with breast } \\
\text { cancer. Rehabilitation Oncology, } 25 \text { (3), 3-16. }\end{array}$ \\
\hline Hardy, S. E. (2009). & $\begin{array}{l}\text { Methylphenidate for the treatment of depressive symptoms, } \\
\text { including fatigue and apathy, in medically ill older adults and } \\
\text { terminally ill adults. American Journal of Geriatric Pharmaco- } \\
\text { therapy, } 7 \text { (1), 34-59. }\end{array}$ \\
\hline $\begin{array}{l}\text { Jacobsen, P. B.; Donovan, K. A.; Vadaparampil, S. T. \& } \\
\text { Small, B. J. (2007). }\end{array}$ & $\begin{array}{l}\text { Systematic review and meta-analysis of psychological and } \\
\text { activity-based interventions for cancer-related fatigue. Health } \\
\text { Psychology, 26 (6), 660-667. }\end{array}$ \\
\hline Kimel, M.; Leidy, N. K.; Mannix, S. \& Dixon, J. (2008). & $\begin{array}{l}\text { Does epoetin alfa improve health-related quality of life in } \\
\text { chronically ill patients with anemia: summary of trials of } \\
\text { cancer, HIV/AIDS, and chronic kidney disease. Value in Health, } \\
11 \text { (1), 57-75. }\end{array}$ \\
\hline Kuchinski, A.; Reading, M. \& Lash, A. A. (2009). & $\begin{array}{l}\text { Treatment-related fatigue and exercise in patients with } \\
\text { cancer: a systematic review. MEDSURG Nursing, } 18 \text { (3), } \\
\text { 174-180. }\end{array}$ \\
\hline $\begin{array}{l}\text { Lotfi-Jam, K.; Carey, M.; Jefford, M.; Schofield, P.; } \\
\text { Charleson, C. \& Aranda, S. (2008). }\end{array}$ & $\begin{array}{l}\text { Nonpharmacologic strategies for managing common } \\
\text { chemotherapy adverse effects: a systematic review. Journal } \\
\text { of Clinical Oncology, } 26 \text { (34), 5618-5629. }\end{array}$ \\
\hline Markes, M.; Brockow, T. \& Resch, K.-L. (2006). & $\begin{array}{l}\text { Exercise for women receiving adjuvant therapy for breast } \\
\text { cancer. Cochrane Database of Systematic Reviews: Reviews } \\
2006 \text { Issue } 4 \text { John Wiley \& Sons, Ltd Chichester, UK DOI: } \\
\text { 10.1002/14651858.CD005001.pub2. }\end{array}$ \\
\hline
\end{tabular}


Tabelle 3: Nach Durchsicht der Volltexte ausgeschlossene Literatur. (Fortsetzung)

\begin{tabular}{|c|c|}
\hline $\begin{array}{l}\text { Minton, O.; Richardson, A.; Sharpe, M.; Hotopf, M. \& Stone, P. } \\
\text { (2010). }\end{array}$ & $\begin{array}{l}\text { Drug therapy for the management of cancer-related fatigue. } \\
\text { Cochrane Database of Systematic Reviews 2010, Issue 7. Art. } \\
\text { No.: CD006704. DOI: } 10.1002 / 14651858 . C D 006704 \text {.pub3. }\end{array}$ \\
\hline $\begin{array}{l}\text { Minton, O.; Richardson, A.; Sharpe, M.; Hotopf, M. \& } \\
\text { Stone, P. C. (2011). }\end{array}$ & $\begin{array}{l}\text { Psychostimulants for the management of cancer-related } \\
\text { fatigue: a systematic review and meta-analysis. Journal of } \\
\text { Pain and Symptom Management, } 41 \text { (4), 761-767. }\end{array}$ \\
\hline Mock V. (2004). & $\begin{array}{l}\text { Evidence-based treatment for cancer-related fatigue. Journal } \\
\text { of the National Cancer Institute Monographs 32, 112-118. }\end{array}$ \\
\hline $\begin{array}{l}\text { Oldervoll, L. M.; Kaasa, S.; Hjermstad, M. J.; Lund, J. A. \& } \\
\text { Loge, J. H. (2004). }\end{array}$ & $\begin{array}{l}\text { Physical exercise results in the improved subjective well-being } \\
\text { of a few or is effective rehabilitation for all cancer patients? } \\
\text { European Journal of Cancer, } 40(7), 951-962 \text {. }\end{array}$ \\
\hline $\begin{array}{l}\text { Peuckmann-Post, V.; Elsner, F.; Krumm, N.; Trottenberg, P. \& } \\
\text { Radbruch, L. (2010). }\end{array}$ & $\begin{array}{l}\text { Pharmacological treatments for fatigue associated with } \\
\text { palliative care. Cochrane Database of Systematic Reviews: } \\
\text { Reviews } 2010 \text { Issue } 11 \text { John Wiley \& Sons, Ltd Chichester, UK } \\
\text { DOI: } 10.1002 / 14651858 . C D 006788 . p u b 2 .\end{array}$ \\
\hline $\begin{array}{l}\text { Schmitz, K. H.; Holtzman, J.; Courneya, K. S.; Mâsse, L. C.; } \\
\text { Duval, S. \& Kane, R. (2005). }\end{array}$ & $\begin{array}{l}\text { Controlled physical activity trials in cancer survivors: a } \\
\text { systematic review and meta-analysis. Cancer Epidemiology, } \\
\text { Biomarkers \& Prevention, } 14 \text { (7), 1588-1595. }\end{array}$ \\
\hline Servaes, P.; Verhagen, C. \& Bleijenberg G. (2002). & $\begin{array}{l}\text { Fatigue in cancer patients during and after treatment: } \\
\text { prevalence, correlates and interventions. European Journal } \\
\text { of Cancer, } 38 \text { (1), } 27-43 \text {. }\end{array}$ \\
\hline Silver, J. K. \& Gilchrist, L. S. (2011). & $\begin{array}{l}\text { Cancer rehabilitation with a focus on evidence-based } \\
\text { outpatient physical and occupational therapy interventions. } \\
\text { American Journal of Physical Medicine \& Rehabilitation, } \\
90 \text { (5 Suppl 1), S5-15. }\end{array}$ \\
\hline Taillibert, S.; Laigle-Donadey, F. \& Sanson, M. (2004). & $\begin{array}{l}\text { Palliative care in patients with primary brain tumors. } \\
\text { Current Opinion in Oncology, } 16(6), 587-592 .\end{array}$ \\
\hline
\end{tabular}

sicht der Volltexte ausgeschlossen, da sie aufgrund späterer, auf bestimmte Interventionen abzielender Arbeiten als überholt gelten mussten. Zwei Arbeiten mit allgemeinen Zielsetzungen wurden jedoch eingeschlossen, da sie speziell Pflegeinterventionen bei Fatigue während der Krebsbehandlung umfassten.

Alle eingeschlossenen Arbeiten waren in englischer Sprache verfasst.

In den meisten Übersichtsarbeiten wurde keine Unterscheidung für berufsgruppenspezifische Verantwortungsbereiche gezogen. Es gab wenige Arbeiten, welche international die Evidenz speziell für Pflegekräfte aufbereitet hatten. Dort waren Interventionen eingeschlossen, die in anderen Reviews in die Kategorien nichtpharmakologische Therapien, psychosoziale Interventionen oder Komplementärtherapien eingeordnet wurden. Ebenso wenig wurden Interventionen unterschieden, die üblicherweise von Sporttherapeuten oder Physiotherapeuten durchgeführt werden. Für die Pflege hat dies den Vorteil, dass Patient(inn)en auf der Grundlage der vorliegenden Evidenz umfassend beraten und geschult werden können. Zudem sind die Kompetenzen von Pflegekräften nicht eindeutig festgelegt und entwickeln sich langfristig weiter, können sich also durchaus auf Bereiche erweitern, die bislang noch außerhalb der Pflege liegen. Lediglich be der Pharmakotherapie ist die Zuordnung in den ärztlichen Verantwortungsbereich eindeutig, daher wurden solche Arbeiten ausgeschlossen.

Die Reviews ließen sich folgenden Themen zuordnen (vgl. Tabelle 4): allgemeine Reviews

- Pflegeinterventionen

- Nichtpharmakologische Interventionen

- psychosoziale Interventionen
- komplementäre und alternative Medizin

spezielle Reviews

- körperliches Training/körperliche Aktivität

- Physio- und Ergotherapie

- Entspannung

- Musiktherapie

- Tanztherapie

- Kunsttherapie

- Akupunktur

- Yoga

- Massage

- chinesische Kräutermedizin.

Die Reviews zu körperlichem Training/ körperlicher Aktivität wurden je nach Zielgruppe weiter unterteilt.

Die wissenschaftliche Qualität der eingeschlossenen Reviews war überwiegend gut (vgl. Tabelle 5). Am häufigsten wurden gemäß AMSTAR die Items 5 «Was a list of studies (included and excluded) provided?» (16×) und 11 «Was 


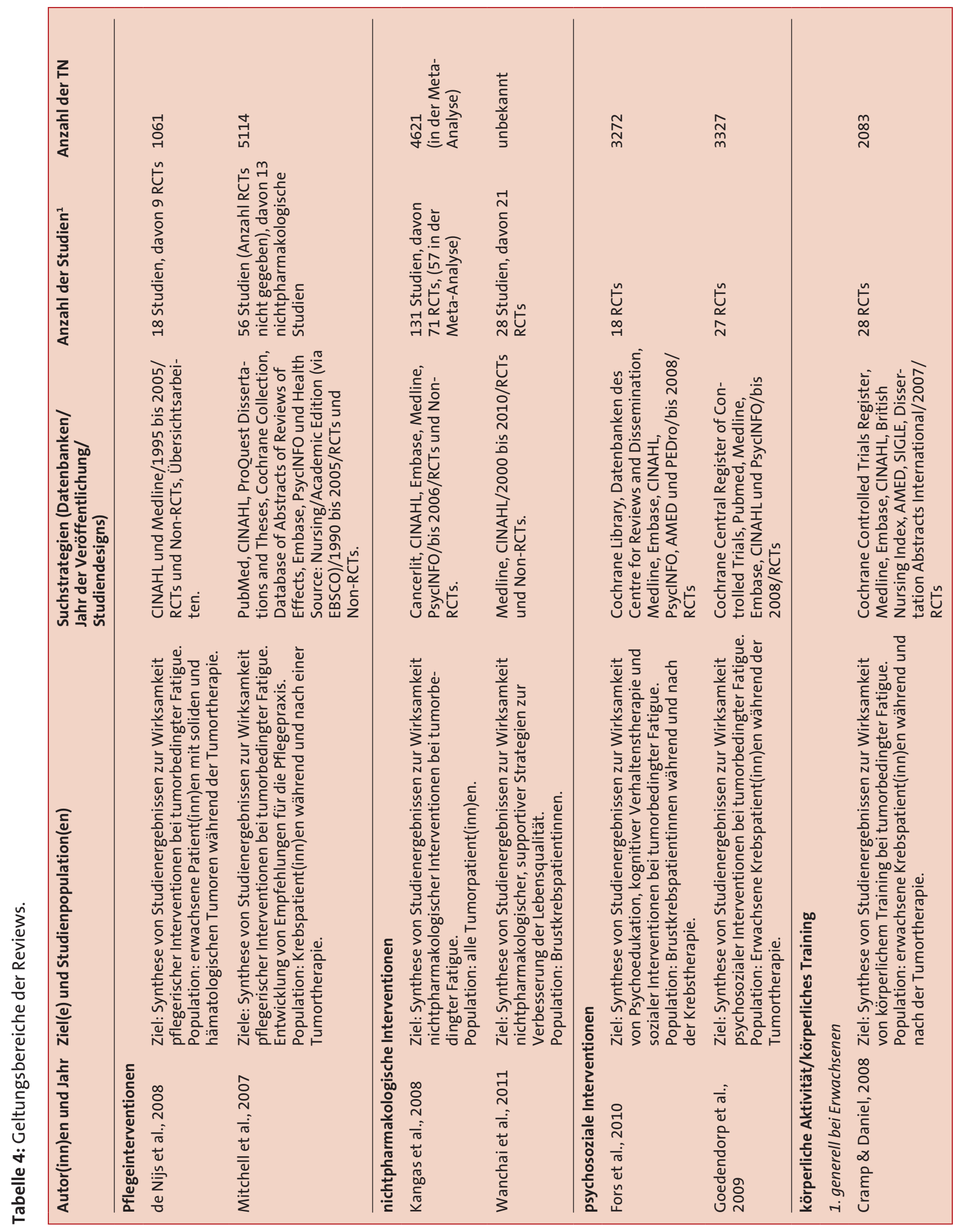




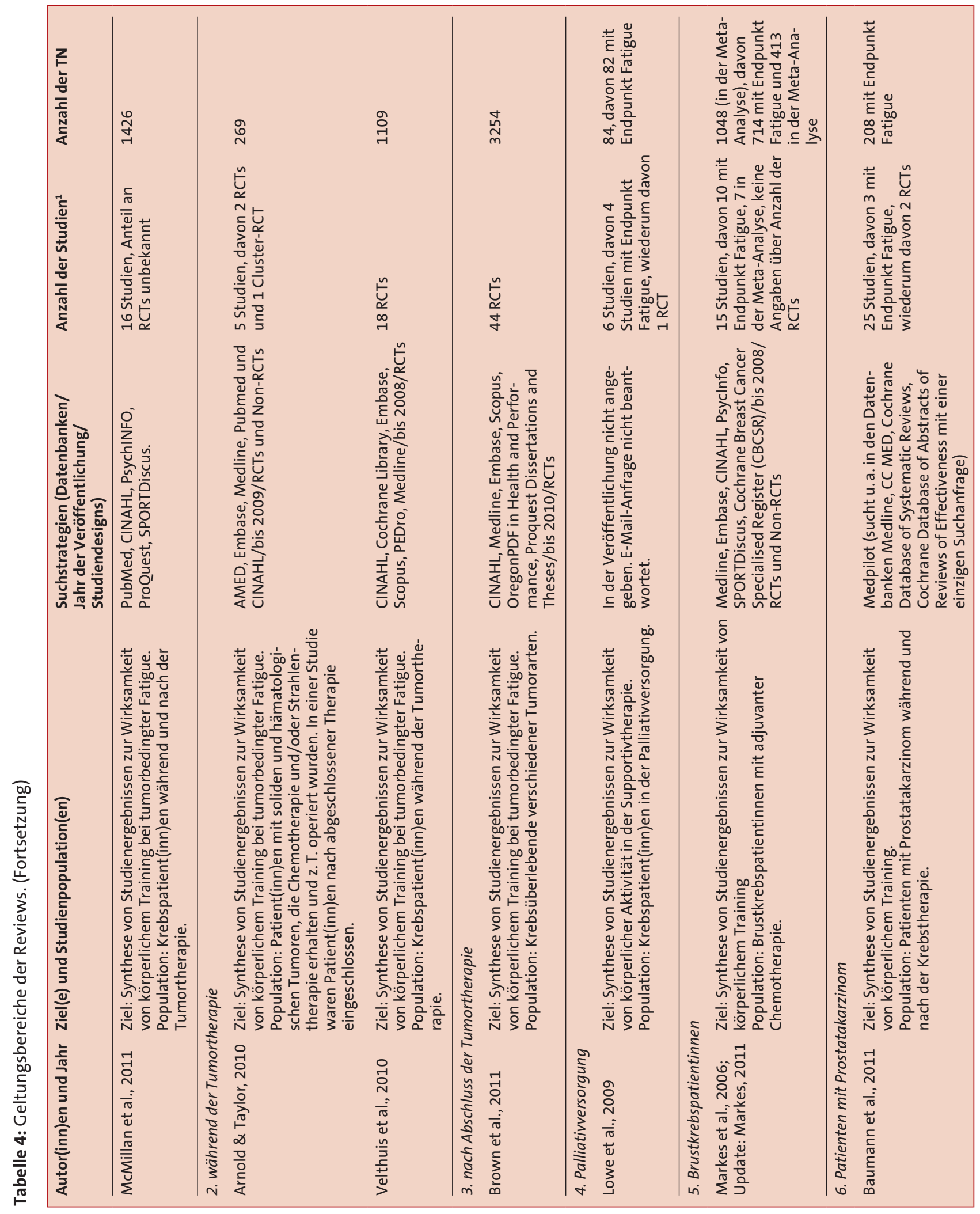




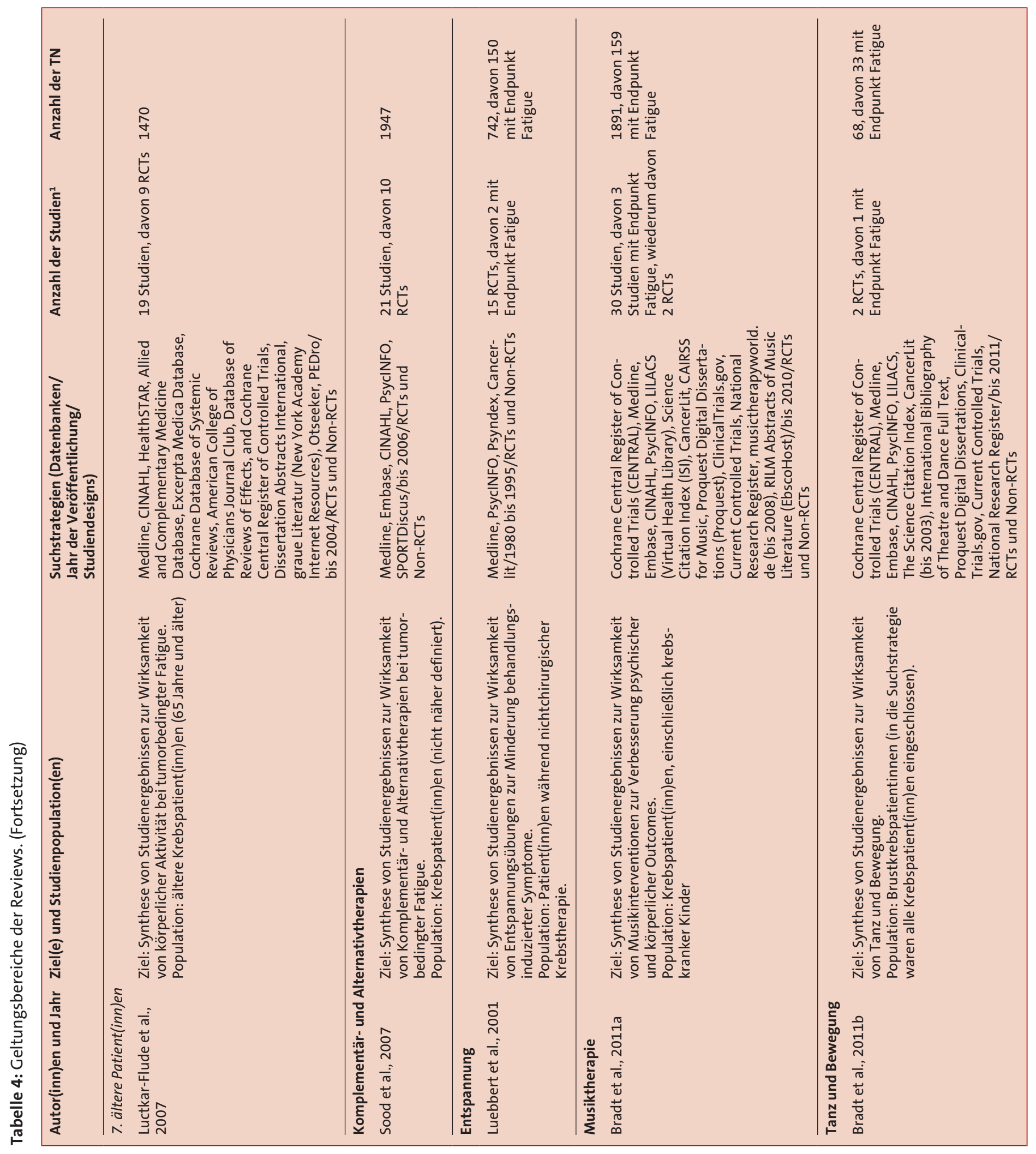




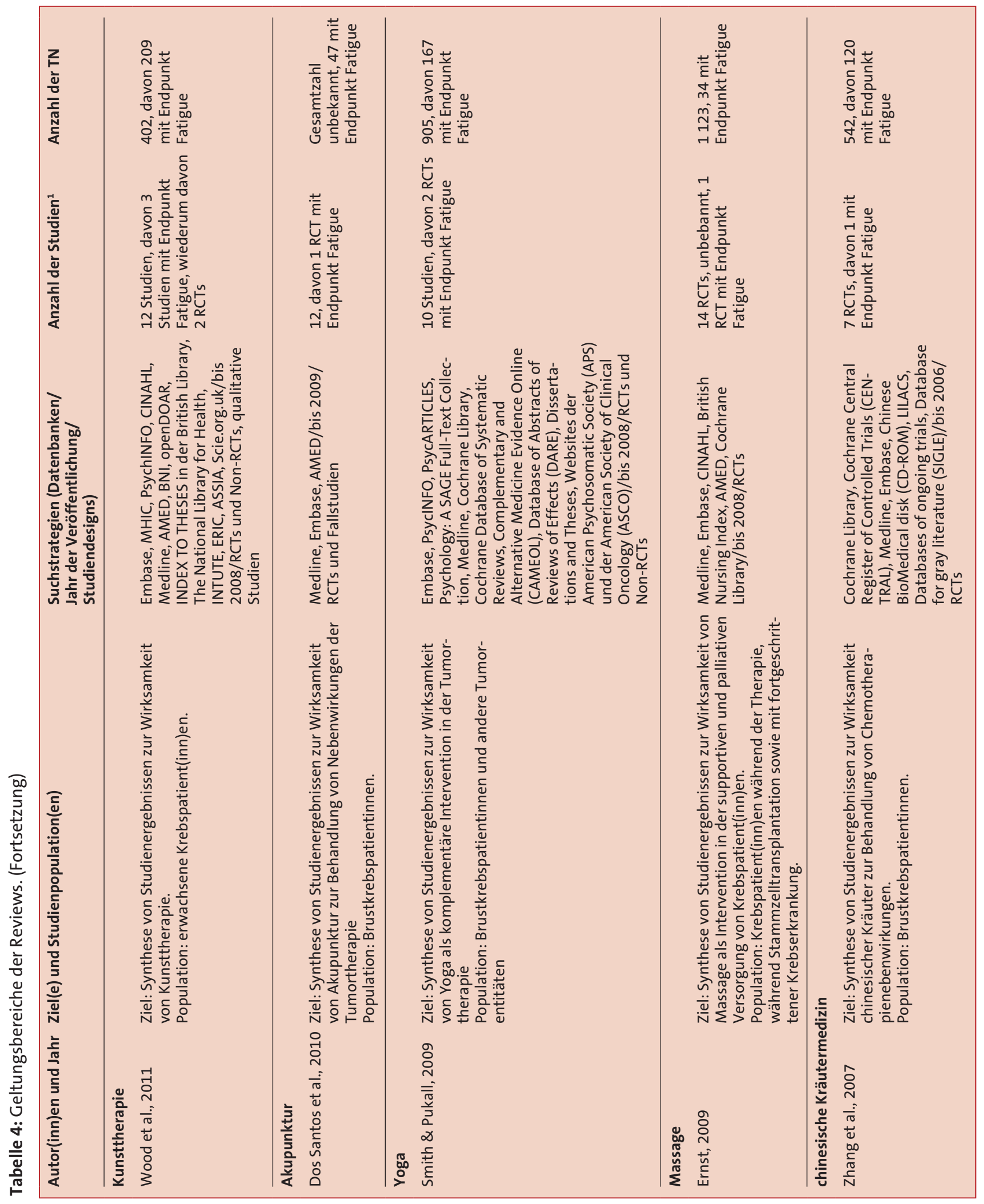


the conflict of interest stated?» (13×) mit «Nein» beantwortet. Item 5 musste häufig mit «Nein» beantwortet werden, weil keine Liste der ausgeschlossenen Studien vorlag. Die eingeschlossenen Studien waren in der Regel gut dokumentiert.

Dieser Overview umfasst 577 Primärstudien, davon sind einige in mehreren Reviews eingeschlossen. Nach Abzug dieser Mehrfacheinschlüsse sowie der pharmakologischen Studien verbleiben 346 Originalarbeiten. Diese Berechnung beruht auf 23 Übersichtsarbeiten, von einem Review standen nicht ausreichend Informationen zur Verfügung (Dos Santos, Hill, Morgan, Smith, Thai, Cheifetz et al., 2010). Überschneidungen traten vor allem zwischen themenverwandten Reviews auf. Die Übersichten zu Pflegeinterventionen, nichtpharmakologischen Interventionen und psychosozialen Interventionen basieren auf insgesamt 235 Originalarbeiten, nach Entfernung von Mehrfacheinschlüssen blieben 169 Studien. Bei den Reviews zu körperlichem Training ist das Verhältnis 176 zu 101 Studien. Zwischen den speziellen Reviews kamen fast keine Überschneidungen vor.

Es wurden zwei systematische Übersichtsarbeiten gefunden, die sich mit Pflegeinterventionen bei Patient(inn)en mit tumorbedingter Fatigue befassten (für alle Ergebnisse: siehe Tabellen 3 und 4). Ein Einschlusskriterium in diesen Arbeiten war, dass Pflegekräfte diese Interventionen durchführten. Die dort eingeschlossenen Interventionen umfassten körperliches Training, Patient(inn)enedukation und Beratung, Symptommanagement, Arzneimitteltherapie, Ablenkung und Entspannung, Schlafförderung, energieerhaltende Maßnahmen, Aktivitätenmanagement, integrative Medizin. Mitchell, Beck, Hood, Moore und Tanner (2007) leiteten aus der Übersicht Empfehlungen für die Praxis ab. Aus beiden Übersichten ist zu schließen, dass körperliches
Training die wichtigste Intervention bei Fatigue ist. Für andere Maßnahmen ist die Evidenz eher schwach ausgeprägt. Wanchai, Armer und Stewart (2011) kommen zwar aus der Pflegeforschung und sprechen in ihrer Arbeit von Pflegeinterventionen, hatten aber allgemeine, nichtpharmakologische Interventionen eingeschlossen. Dieser Review wird daher bei den Interventionen für Brustkrebspatientinnen besprochen. Zwei Arbeiten wurden eingeschlossen, die sich mit nichtpharmakologischen Interventionen befassten: eine umfassende Arbeit, die von allen Reviews die meisten Studien eingeschlossen hatte (Kangas, Bovbjerg und Montgomery, 2008), sowie eine Arbeit von Wanchai und Kolleginnen (2011), die sich auf Brustkrebspatientinnen beschränkte und als Zielparameter Lebensqualität hatte. Hier zeigten sich insbesondere Bewegungsförderung sowie psychosoziale Interventionen wie Patient(inn)enedukation und Beratung als wirksam. Aber auch Schlaftherapie sowie komplementärmedizinische Maßnahmen zeigten in einigen Studien Effekte.

Psychosoziale Interventionen können zu den nichtpharmakologischen Interventionen gezählt werden. Es wurden zwei Reviews eingeschlossen, die psychosoziale Interventionen untersuchten. Zum einen handelt es sich um einen Cochrane-Review, dessen Zielgruppe erwachsene Krebspatient(inn)en während der Tumortherapie waren (Goedendorp, Gielissen, Verhagen \& Blejenberg, 2009), zum anderen um einen Review, der sich auf Rehabilitationsprogramme bei Brustkrebspatientinnen begrenzte (Fors, Bertheussen, Thune, Juvet, Elvsaas, Oldervoll et al., 2011). Insgesamt liegt ausreichend Evidenz für psychosoziale Interventionen mit Effektstärken zwischen 0,17 und 1,07 vor. Jedoch ist die Studienlage für psychosoziale Interventionen in Rehabilitationsprogrammen für Brustkrebspatientinnen eher schwach.
Die beste Evidenz zur Minderung der Fatiguebelastung bei Krebspatient(inn)en liegt für körperliches Training beziehungsweise körperliche Aktivität vor. Es wurden etliche Übersichtsarbeiten zu diesem Thema veröffentlicht. Der Betrag des jeweiligen standardisierten Mittelwertunterschieds (standardised mean difference, SMD) unterschied sich in den verschiedenen Reviews nicht sehr: Die Werte lagen über alle Tumorarten und alle Tumorstadien hinweg bei 0,23 beziehungsweise 0,28. Nach Abschluss der Tumortherapie beziehungsweise bei Krebsüberlebenden liegt die Effektstärke etwas höher ( 0,37 beziehungsweise 0,31) als bei Patient(inn)en während der Tumortherapie $(0,18$ beziehungsweise 0,24). Bezogen auf verschiedene Formen des Trainings zeigte sich der Effekt von Ausdauertraining $(\mathrm{SMD}=0,25)$ als besser belegt als der Effekt von Krafttraining oder einer Kombination aus beiden Trainingsformen, für die es keine statistisch signifikanten Ergebnisse aus Meta-Analysen gibt. Für Patient(inn)en in der Palliativversorgung ist die Studienlage defizitär. Die Ergebnisse zweier Pilotstudien weisen jedoch auf einen möglichen Effekt auch bei dieser Patient(inn)engruppe hin. Brustkrebs ist diejenige Tumorentität, die am besten untersucht wurde. Die Effektstärken des Trainings lagen hier zwischen 0,17 und 0,36. Markes (2011) verwendete etwa zur Hälfte andere Quellen als Grundlage ihrer Meta-Analyse als McMillan und Newhouse (2011), während Cramp und Daniel (2008) für ihre Meta-Analyse die umfassendste Grundlage (16 Studien) verwendeten. Ähnliche Effekte fanden sich auch für Patienten mit Prostatakarzinom, allerdings bei erheblich weniger Studien (SMD =0,32). Für andere Tumorentitäten ist die Studienlage schwach, in einer Meta-Analyse von Patient(inn)en mit anderen Tumorarten als Brustkrebs war die Ef- 


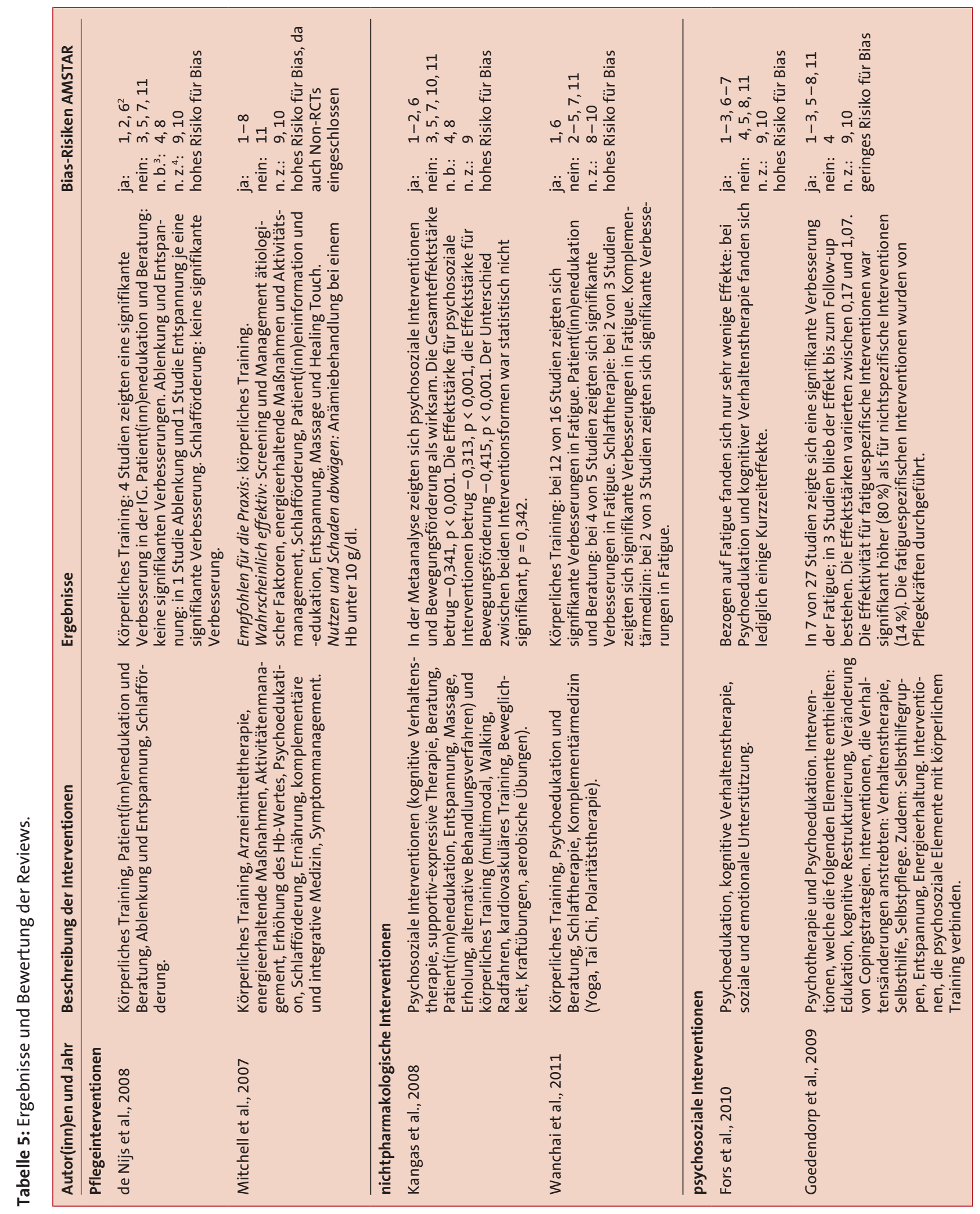




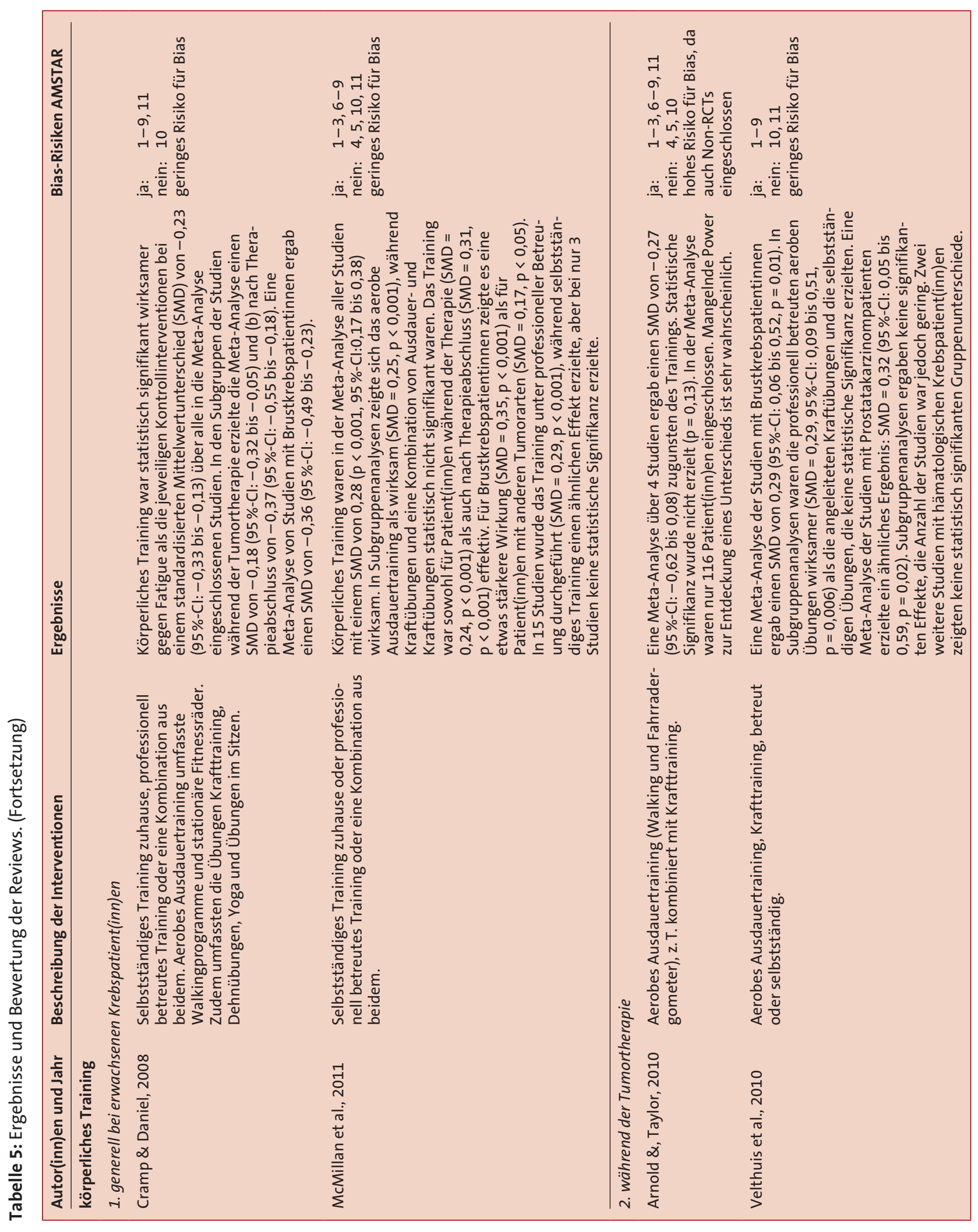




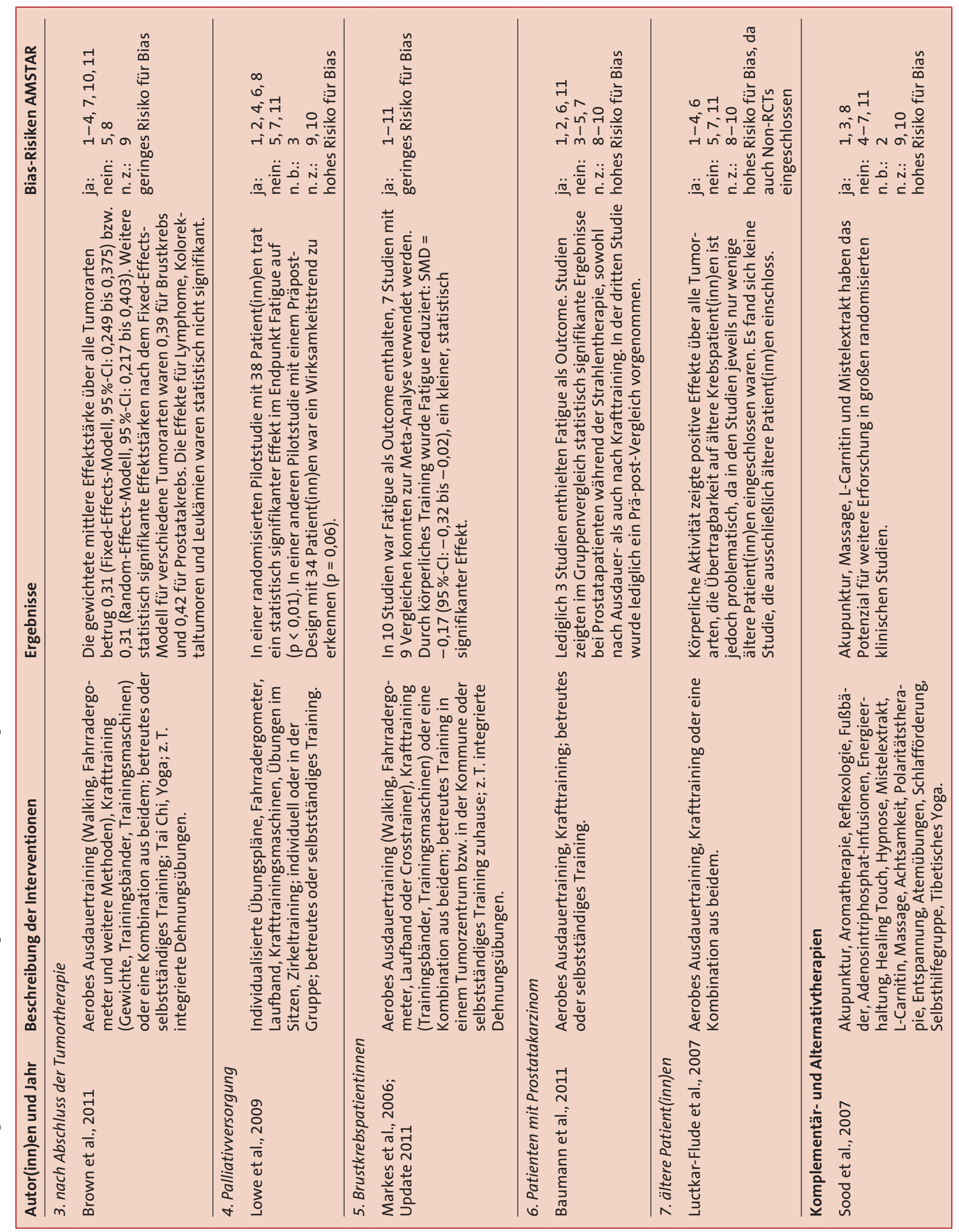




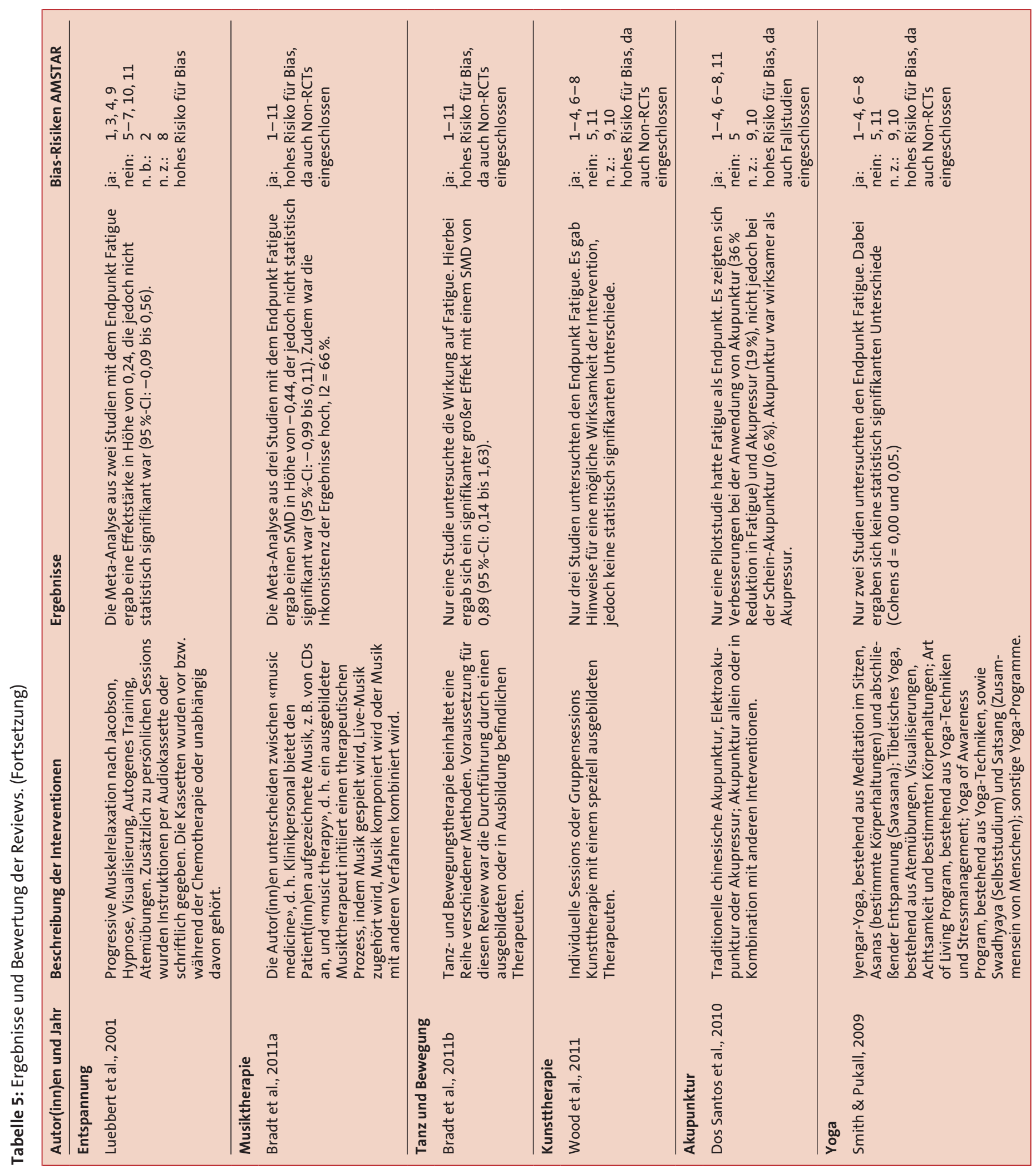


fektstärke gering $(0,17)$. Für die Gruppe der älteren Krebspatient(inn)en wie auch für krebskranke Kinder fand sich keine Evidenz aus systematischen Übersichten.

Für Komplementär- und Alternativtherapien, Interventionen zur Entspannung, Musik-, Tanz- und Kunsttherapie, Massage, Akupunktur, Yoga und chinesische Kräutermedizin war die Evidenz hinsichtlich der Senkung der Fatiguebelastung bei Krebspatient(inn)en sehr schwach. Es gab zwar durch wenige Einzelstudien Hinweise auf eine mögliche Wirksamkeit dieser Maßnahmen, Nachweise in großen randomisierten klinischen Studien stehen jedoch noch aus.

\section{Diskussion}

Diese Übersicht zeigt sehr deutlich, dass vor allem körperliche Bewegung, sei es in Form von Ausdauer- oder Krafttraining oder auch in Form von alltäglicher Bewegung, die Fatiguebelastung von Krebspatient(inn)en vermindern kann. Doch trotz einer hohen Zahl an randomisierten klinischen Studien wie auch systematischen Übersichtsarbeiten ist die Studienlage noch an vielen Stellen defizitär. Die Evidenz für moderates Ausdauertraining ist am stärksten, was aber auch daran liegt, dass es bislang nur relativ wenige Studien über Krafttraining gibt. Des Weiteren wurden die meisten Studien bei Brustkrebspatientinnen und Patienten mit Prostatakarzinom durchgeführt. Bei anderen Tumorentitäten ist die Studienlage eher dünn. Wir finden gute Evidenz für Patient(inn)en während einer kurativen Tumortherapie wie auch für Patient(inn)en nach Therapieabschluss. Nach Therapieabschluss scheint die Wirksamkeit etwas höher zu sein, doch bereits bei Therapiebeginn können Patient(inn)en aus angepasstem körperlichem Training einen
Nutzen ziehen, nicht nur hinsichtlich Fatigue sondern auch sonstiger Nebenwirkungen der Krebstherapie (Dimeo \& Thiel, 2008). Für Patient(inn)en in der Palliativversorgung fehlen noch entsprechende Untersuchungen. Des Weiteren ist die Studienlage gut bei professionell betreuten Bewegungsprogrammen. Schwächer ist die Evidenz jedoch bei selbstständigen Übungen oder einer Kombination aus professioneller Anleitung und selbstständigen Übungen. Da die Patient(inn)en ihre Bewegungsprogramme jedoch nach der Anleitung selbstständig fortführen sollten, um einen langfristigen Erfolg zu erzielen, sind auch hier weitere Studien erforderlich.

Trotz guter Wirksamkeitsnachweise scheinen Krebspatient(inn)en nicht ausreichend über die Vorteile körperlicher Aktivität informiert zu sein. Eine Befragung, an der 317 Patient(inn)en von niedergelassenen Hämatoonkologen teilnahmen, zeigt hier deutlich die Defizite auf (Wittmann, Bernhörster, Vogt \& Banzer, 2011). Die befragten Patient(inn)en fühlten sich sehr gut informiert sowohl über ihre Therapie (57\%) als auch über Therapienebenwirkungen (41\%). Bezüglich des Nutzens der Bewegungstherapie fühlten sich jedoch nur $10 \%$ sehr gut informiert, $48 \%$ waren über den Nutzen überhaupt nicht informiert. Die wichtigste Informationsquelle waren für die Patient(inn)en die Ärzte $(81 \%)$, die Pflegekräfte waren nur für $24 \%$ der Befragten eine Informationsquelle. Hier zeigt sich für die Pflege ein eindeutiger Nachholbedarf. Pflege sollte zukünftig bereits zu Beginn einer Tumorbehandlung über den Nutzen von angepasster, körperlicher Aktivität informieren und die Patient(inn)en zu entsprechenden Tätigkeiten ermuntern. Onkologische Pflegekräfte sollten sich dafür einsetzen, dass die Patient(inn)en bereits während der Tumorbehandlung Bewegungsangebote erhalten und nach 
Therapieabschluss verstärkt Nachsorgesport in Anspruch nehmen.

Das zweite wichtige Arbeitsfeld zur Reduktion der Fatiguebelastung liegt in psychosozialen Interventionen. Besonders interessant ist, dass die Evidenz für von Pflegekräften durchgeführte fatiguespezifische Interventionen deutlich stärker ist als für nichtspezifische Interventionen. Wirksame Komponenten dieser Interventionen waren Psychoedukation, also Information über Fatigue und Fatiguemanagement, Selbstpflege und Copingstrategien, Aktivitätsmanagement, das heißt der Ausgleich zwischen Aktivität und Ruhephasen, sowie emotionale Unterstützung.

Somit erscheint es sinnvoll, körperliche Aktivität und psychosoziale Interventionen $\mathrm{zu}$ multimodalen Interventionen zu verbinden. Dieses Konzept wurde in einem neu entwickelten Selbstmanagementprogramm für Krebspatient(inn)en umgesetzt und steht zur Anwendung bereit (de Vries, Reif, Petermann \& Görres, 2011). Für Patient(inn)en wurde das Programm zur selbstständigen Bearbeitung aufbereitet (Reif, de Vries, Petermann \& Görres, 2011).

Für weitere Interventionsformen ist derzeit die Evidenz schwach, es gibt jedoch vielversprechende Ansätze wie etwa Tanztherapie, die ihre Wirkung möglicherweise über die Bewegungsförderung, aber auch über psychosoziale Komponenten entfalten könnte, sowie Entspannung, Massage oder Akupunktur.

Die medikamentöse Behandlung von Fatigue wurde international in mehreren Studien untersucht. Eine MetaAnalyse kommt zu dem Schluss, dass Psychostimulanzien zu einer Linderung von Fatigue führen können, dass jedoch zur Sicherung dieser Befunde groß angelegte randomisierte Studien erforderlich sind (Minton, Richardson, Sharpe, Hotopf \& Stone, 2011). Auch hämatopoetische Wachstumsfaktoren sind gegen Fatigue wirksam, werden jedoch aufgrund ihres hohen Nebenwirkungspotenzials bei Fatigue nicht mehr empfohlen (Minton, Richardson, Sharpe, Hotopf \& Stone, 2010). In Deutschland sind Psychostimulanzien zur Behandlung der Fatigue derzeit nicht zugelassen; eine erste Studie mit 37 Patient(inn)en bestätigte die internationalen Ergebnisse (Wirz, Nadstawek, Kühn, Vater, Junker \& Wartenberg, 2010). Zurzeit muss die Pharmakotherapie der Fatigue weiteren wissenschaftlichen Studien vorbehalten bleiben und kann nicht allgemein empfohlen werden. Zudem empfiehlt das NCCN in der Fatigueleitlinie, eine medikamentöse Therapie erst nach Ausschöpfung der nichtpharmakologischen Optionen zu erwägen (National Comprehensive Cancer Network, 2012).

Diese Übersichtsarbeit weist einige Limitationen auf. So wurde auf eine Handsuche, eine Suche nach unveröffentlichter Literatur, das Anschreiben von Experten wie auch auf die Suche nach Einzelstudien verzichtet. Da es allgemein und speziell ausgerichtete Übersichten gab, war nicht zu vermeiden, dass sich die in den Reviews eingeschlossenen Studien zum Teil überschnitten. Dies wurde bewusst in Kauf genommen, um insbesondere auch Reviews mit speziellen Interventionen darstellen zu können.

\section{Fazit für die Pflegepraxis}

Krebspatient(inn)en erleben im Verlauf ihrer Erkrankung existenziell belastende und bedrohliche Situationen, durch die sie und ihre Familien stark gefordert werden. In den verschiedenen Krankheits- und Lebensphasen müssen sie ihren Alltag neu gestalten und Strategien zur Krankheitsbewältigung entwickeln. Fatigue ist eine der stärks- ten Belastungen, denen sie vom Zeitpunkt der Diagnosestellung bis Jahre nach Therapieabschluss ausgesetzt sind. Laut Bundesarbeitsgruppe der Leitungen der Weiterbildungsstätten für die Pflege des krebskranken, chronisch kranken Menschen (Bundesarbeitsgruppe der Leitungen der Weiterbildungsstätten für die Pflege des krebskranken, chronisch kranken Menschen (BAGL), 2005) übernehmen onkologisch spezialisierte Pflegende Verantwortung in den Aufgabenbereichen Anleitung, Beratung und Schulung, Beziehungsgestaltung sowie Koordination und Organisation. Eine wesentliche Grundlage hierfür sind gute Kenntnisse aus der Evaluationsforschung. Der vorliegende Überblick stellt erstmals die gesamte Studienlage der Wirkungsforschung für tumorbedingte Fatigue dar.

Es hat sich insbesondere gezeigt, dass individuell angepasste körperliche Aktivität unter Berücksichtigung von Kontraindikationen sehr gut zur Fatiguebewältigung beiträgt. Zudem sollten die Patient(inn)en psychosoziale Unterstützung, möglichst auch über den Krankenhausaufenthalt hinaus, erhalten. Dies sollte bei pflegerischen Interventionen wie auch in der Beratung von Krebspatient(inn)en zukünftig verstärkt berücksichtigt werden. Erst wenn diese gut evaluierten Interventionen ausgeschöpft wurden, können weitere, etwa komplementäre Pflegemaßnahmen empfohlen werden. Die Pflege von Krebspatient(inn)en mit Fatigue ist eine Herausforderung, die eine differenzierte Herangehensweise erfordert: Nicht nur müssen die durch Fatigue verursachten Einschränkungen bei allen pflegerischen Tätigkeiten berücksichtigt werden, die Patient(inn) en sollten auch bei der Entwicklung eines angepassten Selbstmanagements unterstützt und $\mathrm{zu}$ wirksamen Interventionen angeleitet werden. 


\section{Verantwortlichkeiten}

KR: Konzept, Datenerhebung, Studienbewertung, Verfassen des Entwurfs UdV: Datenerhebung, Studienbewertung, Korrekturlesen des Manuskripts FP: Korrekturlesen des Manuskripts

\section{What does really help against} cancer-related fatigue? An overview of systematic reviews

Cancer-related fatigue, a subjective feeling of a distinctive physical, emotional and/or cognitive tiredness, is a common phenomenon in patients with cytotoxic chemotherapy or radiotherapy. Research on cancer-related fatigue has been intensified in the last decade. The objective of this work was to give an overview of the effectiveness of non-pharmacological interventions for cancer-related fatigue. Therefore, the databases of the Cochrane Library, PubMed, CINAHL and DAHTA were searched for systematic reviews. Two persons independently selected the reviews and assessed the methodological quality. We used the measurement tool AMSTAR to assess the methodological quality of each systematic review. The results were summarised in a qualitative synthesis. A total of 24 reviews were included in the analysis. The results show that physical activity and psycho-social support can reduce fatigue in cancer patients. Particularly, nurseled fatigue specific interventions proved to be effective. Currently the evidence for other intervention types is weak; however there exist some promising approaches. Despite a large number of randomised clinical trials as well as systematic reviews the evidence base is still deficient in many respects. The results can be used by nurses in clinical care, counselling and education of cancer patients.

Key words: fatigue, cancer, systematic review, oncology nursing, nursing research

\section{Literatur}

Al-Majid, S.; McCarthy, D. O. (2001) Cancer-induced fatigue and skeletal muscle wasting: the role of exercise. Biological Research for Nursing, 2 (3), 186 - 97.

Arnold, M.; Taylor, N. F. (2010). Does exercise reduce cancer-related fatigue in hospitalised oncology patients? A systematic review. Onkologie, 33 (11), 625-30.

Baumann, F. T.; Zopf, E. M.; Bloch, W. (2012). Clinical exercise interventions in prostate cancer patients a systematic review of randomized controlled trials. Supportive Care in Cancer, 20 (2), 221 - 33.

Becker, L. A.; Oxman, A. D. (2011). Chapter 22: Overviews of reviews. In: Higgins, J. P.T.; Green, S. (eds.). Cochrane Handbook for Systematic Reviews of Interventions Version 5.1.0 (updated March 2011). The Cochrane Collaboration. www.cochrane-handbook. org. [12.06.2012].

Berger, A. M.; Wielgus, K.; Hertzog, M.; Fischer, P.; Farr, L. (2010). Patterns of circadian activity rhythms and their relationships with fatigue and anxiety/depression in women treated with breast cancer adjuvant chemotherapy. Supportive Care in Cancer, 18 (1), 105 - 114.

Bradt, J.; Dileo, C.; Grocke, D.; Magill, L. (2011a). Music interventions for improving psychological and physical outcomes in cancer patients.
Cochrane Database of Systematic Reviews 2011, Issue 8. Art. No.: CD006911. DOI: 10.1002/14651858. CD006911.pub2.

Bradt, J.; Goodill S. W.; Dileo, C. (2011b). Dance/movement therapy for improving psychological and physical outcomes in cancer patients. Cochrane Database of Systematic Reviews 2011, Issue 10. Art. No.: CD007103. DOI: 10.1002/14651858. CD007103.pub2.

Brown, J. C.; Huedo-Medina, T. B.; Pescatello, L. S.; Pescatello, S. M.; Ferrer, R. A.; Johnson, B. T. (2011). Efficacy of exercise interventions in modulating cancer-related fatigue among adult cancer survivors: a meta-analysis. Cancer Epidemiology, Biomarkers \& Prevention, 20 (1), 123 - 133.

Bundesarbeitsgruppe der Leitungen der Weiterbildungsstätten für die Pflege des krebskranken, chronisch kranken Menschen (BAGL) (2005). Aufgabenprofil Pflegender mit vertiefter Kompetenz in der Pflege krebskranker Menschen. http:// www.kok-krebsgesellschaft.de/index.php/arbeitsgruppen/bagl.html [15.04.2012].

Cramp, F.; Daniel, J. (2008). Exercise for the management of cancer-related fatigue in adults. Cochrane Database of Systematic Reviews 2008, Issue 2. Art. No.: CD006145. DOI: 10.1002/14651858.CD006145.pub2. de Nijs, E. J.; Ros, W.; Grijpdonck, M. H. (2008). Nursing intervention for fatigue during the treatment for cancer. Cancer Nursing, 31 (3), $191-208$

de Vries, U.; Reif, K.; Petermann, F. (2011a). Tumorbedingte Fatigue und ihre psychosozialen Belastungen. Der Internist, 52 (11), $1317-1324$.

de Vries, U.; Reif, K.; Petermann, F. (2011b). Tumorbedingte Fatigue: Psychosoziale Hilfen. Der Onkologe, 17 (9), 853 - 860. 
de Vries, U.; Reif, K.; Petermann, F.; Görres, S. (2011). Fatigue individuell bewältigen (FIBS). Schulungsmanual und Selbstmanagementprogramm für Menschen mit Krebs. Bern: Hans Huber.

de Vries, U.; Reif, K.; Stuhldreher, N.; Petermann, F.; Görres, S. (2009). Tumorbedingte Fatigue. Zeitschrift für Gesundheitspsychologie, 17 (4), $170-184$.

Dimeo, F. C.; Thiel, E. (2008). Körperliche Aktivität und Sport bei Krebspatienten. Der Onkologe, 14, 31 - 37.

Dos Santos, S.; Hill, N.; Morgan, A.; Smith, J.; Thai, C.; Cheifetz, O. (2010). Acupuncture for treating common side effects associated with breast cancer treatment: a systematic review. Medical Acupuncture, 22 (2), 81 - 97.

Ernst, E. (2009). Massage therapy for cancer palliation and supportive care: a systematic review of randomised clinical trials. Supportive Care in Cancer, 17 (4), 333 - 337.

Fors, E. A.; Bertheussen, G. F.; Thune, I.; Juvet, L. K.; Elvsaas, I. K.; Oldervoll, L.; Anker, G.; Falkmer, U.; Lundgren, S.; Leivseth, G. (2011). Psychosocial interventions as part of breast cancer rehabilitation programs? Results from a systematic review. Psycho-Oncology, 20 (9), 909-18.

Goedendorp, M. M.; Gielissen, M. F.; Verhagen, C. A. \& Bleijenberg, G. (2009). Psychosocial interventions for reducing fatigue during cancer treatment in adults. Cochrane Database of Systematic Reviews 2009, Issue 1. Art. No.: CD006953. DOI: 10.1002/14651858.CD006953.pub2.

Hofman, M.; Ryan, J. L.; Figueroa-Moseley, C. D.; Jean-Pierre, P.; Morrow, G. R. (2007). Cancer-related fatigue: the scale of the problem. Oncologist, 12 (Suppl 1), 4-10.

Kangas, M.; Bovbjerg, D. H.; Montgomery, G. H. (2008). Cancer-related fatigue: a systematic and meta-analyt- ic review of non-pharmacological therapies for cancer patients. Psychological Bulletin, 134(5), 700 - 741. Liberati, A.; Altman, D. G.; Tetzlaff, J.; Mulrow, C.; Gøtzsche, P.C.; Ioannidis, J. P. A.; Clarke, M.; Devereaux, P. J.; Kleijnen, J.; Moher, D. (2009). The PRISMA Statement for Reporting Systematic Reviews and MetaAnalyses of Studies That Evaluate Health Care Interventions: Explanation and Elaboration. British Medical Journal, 339: b2700, doi: 10.1136/bmj.b2700.

Lowe, S. S.; Watanabe, S. M.; Courneya, K. S. (2009). Physical activity as a supportive care intervention in palliative cancer patients: a systematic review. Journal of Supportive Oncology, 7 (1), 27 - 34.

Luctkar-Flude, M. F.; Groll, D. L.; Tranmer, J. E.; Woodend, K. (2007). Fatigue and physical activity in older adults with cancer: a systematic review of the literature. Cancer Nursing, 30 (5), E35 - E45.

Luebbert, K.; Dahme, B.; Hasenbring, M. (2001). The effectiveness of relaxation training in reducing treatment-related symptoms and improving emotional adjustment in acute non-surgical cancer treatment: a meta-analytical review. Psycho-Oncology, 10 (6), 490 - 502.

Markes, M. (2011). Exercise for women receiving adjuvant therapy of breastcancer: a systematic review. Dissertation. 2011. URN:urn:nbn:de:kobv: 83-opus-29042. [09.01.2012].

Markes, M.; Brockow, T.; Resch, K.-L. (2006). Exercise for women receiving adjuvant therapy for breast cancer. Cochrane Database of Systematic Reviews: Reviews 2006 Issue 4 John Wiley \& Sons, Ltd Chichester, UK DOI: 10.1002/14651858. CD005001.pub2.

McMillan, E. M.; Newhouse, I. J. (2011). Exercise is an effective treatment modality for reducing cancer- related fatigue and improving physical capacity in cancer patients and survivors: a meta-analysis. Applied Physiology, Nutrition, and Metabolism, 36 (6), 892 - 903.

Minton, O.; Richardson, A.; Sharpe, M.; Hotopf, M.; Stone, P. (2010). Drug therapy for the management of cancer-related fatigue. Cochrane Database of Systematic Reviews 2010, Issue 7. Art. No.: CD006704. DOI: 10.1002/14651858.CD006704. pub3.

Minton, O.; Richardson, A.; Sharpe, M.; Hotopf, M.; Stone, P. C. (2011). Psychostimulants for the management of cancer-related fatigue: a systematic review and meta-analysis. Journal of Pain and Symptom Management, 41 (4), 761 - 767.

Mitchell, S. A.; Beck, S. L.; Hood, L. E.; Moore, K.; Tanner, E. R. (2007). Putting evidence into practice: evidence-based interventions for fatigue during and following cancer and its treatment. Clinical Journal of Oncology Nursing, 11 (1), 99-113.

National Comprehensive Cancer Network (NCCN). Clinical Practice Guidelines in Oncology. CancerRelated Fatigue. NCCN Guidelines Version 1.2012. http://www.nccn. org/professionals/physician_gls/ pdf/fatigue.pdf [20.04.2012].

Patarca-Montero, R. (Hrsg.) (2004). Handbook of cancer related fatigue. Binghamton: Haworth Medical Press.

Reif, K. (2012). Tumorbedingte Fatigue. Forum Onkologische Pflege, 2 (1), $44-53$.

Reif, K.; de Vries, U.; Petermann, F.; Görres, S. (2010). Chronische Fatigue bei Krebspatienten. Medizinische Klinik, 105, 779-786.

Reif, K.; de Vries, U.; Petermann, F.; Görres, S. (2011). Wege aus der Erschöpfung - Alltagshilfen für Krebspatienten. Bern: Hans Huber. 
Reif, K.; de Vries, U.; Petermann, F.; Smith, V.; Devane, D.; Begley, C. M.; Görres, S. (2012). A patient education program is effective in reducing cancer-related fatigue: A multicentre randomised two-group waiting-list controlled intervention trial. European journal of oncology nursing. Article in Press DOI: 10.1016/j.ejon.2012.07.002.

Shea, B. J.; Bouter, L. M.; Peterson, J.; Boers, M.; Andersson, N.; Ortiz, Z.; Ramsay, T.; Bai, A.; Shukla, V. K.; Grimshaw, J. M. (2007a). External validation of a measurement tool to assess systematic reviews (AMSTAR). PLoS One, 2 (12), e1350, doi:10.1371/journal.pone. 0001350.

Shea, B. J.; Grimshaw, J. M.; Wells, G. A.; Boers, M.; Andersson, N.; Hamel, C.; Porter, A. C.; Tugwell, P.; Moher, D.; Bouter, L. M. (2007b). Development of AMSTAR: a measurement tool to assess the methodological quality of systematic reviews. BMC Medical Research Methodology, 7 (10), doi:10.1186/1471-2288-7-10.

Shea, B. J.; Hamel, C.; Wells, G. A.; Bouter, L. M.; Kristjansson, E.; Grimshaw, J.; Henry, D. A.; Boers, M. (2009). AMSTAR is a reliable and valid measurement tool to assess the methodological quality of systematic reviews. Journal of Clinical Epidemiology, 62 (10), 1013 - 1020.

Smith, K. B.; Pukall C. F. (2009). An evidence-based review of yoga as a complementary intervention for patients with cancer. Psycho-Oncology, 18 (5), $465-475$.
Clarke, M. (2011). Methodology in conducting a systematic review of systematic reviews of healthcare interventions. BMC Medical Research Methodology, 11 (15), doi: 10.1186/1471-2288-11-15.

Sood, A.; Barton, D. L.; Bauer, B. A.; Loprinzi, C. L. (2007). A critical review of complementary therapies for cancer-related fatigue. Integrative Cancer Therapies, 6 (1), 8-13.

Stone, P.; Richards, M.; A'Hern, R.; Hardy, J. (2000). A study to investigate the prevalence, severity and correlates of fatigue among patients with cancer in comparison with a control group of volunteers without cancer. Annals of Oncology, 11, $561-567$.

Stuhldreher, N.; Reif, K.; de Vries, U.; Görres, S.; Petermann, F. (2008). Development and evaluation of a cancer-related fatigue patient education program: protocol of a randomized controlled trial. BMC Nursing, 7 (12), doi:10.1186/14726955-7-12.

Velthuis, M. J.; Agasi-Idenburg, S. C.; Aufdemkampe, G.; Wittink, H. M. (2010). The effect of physical exercise on cancer-related fatigue during cancer treatment: a meta-analysis of randomised controlled trials. Clinical Oncology (The Royal College of Radiologists), 22(3), 208-221. Wanchai, A.; Armer, J. M.; Stewart, B. R. (2011). Nonpharmacologic supportive strategies to promote quality of life in patients experiencing can- cer-related fatigue: a systematic review. Clinical Journal of Oncology Nursing, 15 (2), 203 - 214.

Wirz, S.; Nadstawek, J.; Kühn, K. U.; Vater, S.; Junker, U.; Wartenberg, H. C. (2010). Modafinil zur Behandlung der Tumorfatigue. Eine Interventionsstudie. Schmerz, 24 (6), $587-595$.

Wittmann, N.; Bernhörster, M.; Vogt, L.; Banzer, W. (2011). Körperliche Aktivität bei Tumorerkrankungen Was weiß der Patient? Deutsche Zeitschrift für Sportmedizin, 62 (5), 116- 119.

Wood, M. J.; Molassiotis, A.; Payne, S. (2011). What research evidence is there for the use of art therapy in the management of symptoms in adults with cancer? A systematic review. Psycho-Oncology, 20 (2), 135 - 145.

Zhang, M.; Liu, X.; Li, J.; He, L.; Tripathy, D. (2007). Chinese medicinal herbs to treat the side-effects of chemotherapy in breast cancer patients. Cochrane Database of Systematic Reviews 2007, Issue 2. Art. No.: CD004921. D

\section{Korrespondenzadresse}

Prof. Dr. Karl Reif

Universität Bremen

Institut für Public Health und

Pflegeforschung

Grazer Str. 4

DE-28359 Bremen

Tel.: +49 0421 218-68960

karlreif@uni-bremen.de 